\title{
Advances on Testing C-Planarity of Embedded Flat Clustered Graphs ${ }^{\star}$
}

\author{
Markus Chimani ${ }^{1}$, Giuseppe Di Battista ${ }^{2}$, Fabrizio Frati ${ }^{3}$, and Karsten Klein ${ }^{3}$ \\ 1 Theoretical Computer Science, University Osnabrück, Germany \\ markus.chimani@uni-osnabrueck. de \\ 2 Dipartimento di Ingegneria, University Roma Tre, Italy \\ gdbedia.uniroma3.it \\ 3 School of Information Technologies, The University of Sydney, Australia \\ \{fabrizio.frati, karsten.klein\}asydney.edu.au
}

\begin{abstract}
We show a polynomial-time algorithm for testing $c$-planarity of embedded flat clustered graphs with at most two vertices per cluster on each face.
\end{abstract}

\section{Introduction}

A clustered graph $C(G, T)$ consists of a graph $G(V, E)$, called underlying graph, and of a rooted tree $T$, called inclusion tree, representing a cluster hierarchy on $V$. The vertices in $V$ are the leaves of $T$, and the inner nodes of $T$, except for the root, are called clusters. The vertices that are descendants of a cluster $\alpha$ in T belong to $\alpha$ or are in $\alpha$. A c-planar drawing of $C$ is a planar drawing of $G$ together with a representation of each cluster $\alpha$ as a simple connected region $R_{\alpha}$ enclosing all and only the vertices that are in $\alpha$; further, the boundaries of no two such regions $R_{\alpha}$ and $R_{\beta}$ intersect; finally, only the edges connecting vertices in $\alpha$ to vertices not in $\alpha$ cross the boundary of $R_{\alpha}$, and each does so only once. A clustered graph is $c$-planar if it admits a $c$-planar drawing.

Clustered graphs find numerous applications in computer science [22], thus theoretical questions on clustered graphs have been deeply investigated. From the visualization perspective, the most intriguing question is to determine the complexity of testing $c$ planarity of clustered graphs. Unlike for other planarity variants [21], like upward planarity [14] and partial embedding planarity [1], the complexity of testing c-planarity remains unknown since the problem was posed nearly two decades ago [13].

Polynomial-time algorithms to test the $c$-planarity of a clustered graph $C$ are known if $C$ belongs to special classes of clustered graphs $[7,-11,13,15,16,18,19$, including $c$ connected clustered graphs, that are clustered graphs $C(G, T)$ in which, for each cluster $\alpha$, the subgraph $G[\alpha]$ of $G$ induced by the vertices in $\alpha$ is connected [8 10 13]. Effective ILP formulations and FPT algorithms for testing $c$-planarity have been presented [5,6]. Generalizations of the $c$-planarity testing problem have also been considered [2, 12].

An important variant of the $c$-planarity testing problem is the one in which the clustered graph $C(G, T)$ is flat and embedded. That is, every cluster is a child of the root of $T$ and a planar embedding for $G$ (an order of the edges incident to each vertex) is fixed

^ Research partially supported by the Australian Research Council (grant DE140100708).

C. Duncan and A. Symvonis (Eds.): GD 2014, LNCS 8871, pp. 416 4272014.

(c) Springer-Verlag Berlin Heidelberg 2014 
in advance; then, the $c$-planarity testing problem asks whether a $c$-planar drawing exists in which $G$ has the prescribed planar embedding. This setting can be highly regarded for several reasons. First, several NP-hard graph drawing problems are polynomialtime solvable in the fixed embedding scenario, e.g., upward planarity testing [3, 14] and bend minimization in orthogonal drawings [14,23]. Second, testing c-planarity of embedded flat clustered graphs generalizes testing $c$-planarity of triconnected flat clustered graphs. Third, testing $c$-planarity of embedded flat clustered graphs is strongly related to a seemingly different problem, that we call planar set of spanning trees in topological multigraphs (PSSTTM): Given a non-planar topological multigraph $A$ with $k$ connected components $A_{1}, \ldots, A_{k}$, do spanning trees $S_{1}, \ldots, S_{k}$ of $A_{1}, \ldots, A_{k}$ exist such that no two edges in $\bigcup_{i} S_{i}$ cross? Starting from an embedded flat clustered graph $C(G, T)$, an instance $A$ of the PSSTTM problem can be constructed that admits a solution if and only if $C(G, T)$ is $c$-planar: $A$ is composed of the edges that can be inserted inside the faces of $G$ between vertices of the same cluster, where each cluster defines a multigraph $A_{i}$. The PSSTTM problem is NP-hard, even if $k=1$ [20].

Testing $c$-planarity of an embedded flat clustered graph $C(G, T)$ is a polynomialtime solvable problem if $G$ has no face with more than five vertices and, more in general, if $C$ is a single-conflict clustered graph [11], i.e., the instance $A$ of the PSSTTM problem associated with $C$ is such that each edge has at most one crossing. A polynomial-time algorithm is also known for testing $c$-planarity of embedded flat clustered graphs such that the graph induced by each cluster has at most two connected components [17]. Finally, the $c$-planarity of clustered cycles with at most three clusters [9] or with each cluster containing at most three vertices [19] can be tested in polynomial time.

Our Contribution. In this paper we show how to test $c$-planarity in polynomial time for embedded flat clustered graphs $C(G, T)$ such that at most two vertices of each cluster are incident to any face of $G$. While this setting might seem unnatural at a first glance, its study led to a deep (in our opinion) exploration of some combinatorial properties of highly non-planar topological graphs. Namely, every instance $A$ of the PSSTTM problem arising from our setting is such that there exists no sequence $e_{1}, e_{2}, \ldots, e_{h}$ of edges in $A$ with $e_{1}$ and $e_{h}$ in the same connected component of $A$ and with $e_{i}$ crossing $e_{i+1}$, for every $1 \leq i \leq h-1$; these instances might contain a quadratic number of crossings, which is not the case for single-conflict clustered graphs [11]. Within our setting, performing all the "trivial local" tests and simplifications results in the rise of nice global structures, called $\alpha$-donuts, whose study was interesting to us.

Refer to the full version of the paper [4] for complete proofs.

\section{Saturators, Con-Edges, and Spanning Trees}

A natural approach to test $c$-planarity of a clustered graph $C(G(V, E), T)$ is to search for a saturator for $C$. A set $S \subseteq V \times V$ is a saturator for $C$ if $C^{\prime}\left(G^{\prime}(V, E \cup S), T\right)$ is a $c$-connected $c$-planar clustered graph. Determining the existence of a saturator for $C$ is equivalent to testing the $c$-planarity of $C$ [13]. Thus, the core of the problem consists of determining $S$ so that $G^{\prime}[\alpha]$ is connected, for each $\alpha \in T$, and so that $G^{\prime}$ is planar. For embedded flat clustered graphs (see Fig. 1 a)), the problem of finding saturators 


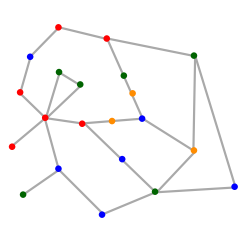

(a)

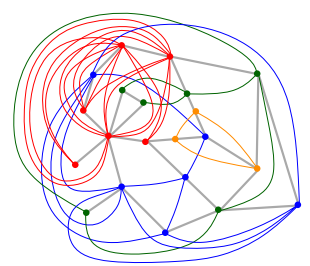

(b)

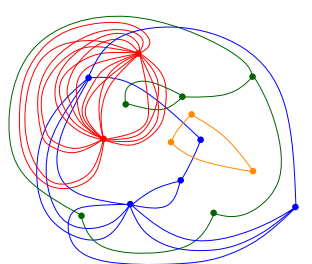

(c)

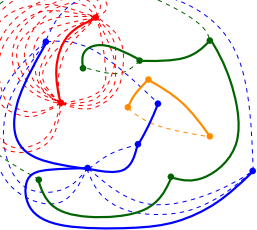

(d)

Fig. 1. (a) A clustered graph $C$. (b) Con-edges in $C$. (c) Multigraph $A$. (d) A planar set $S$ of spanning trees for $A$. Edges in $S$ are thick and solid, while edges in $A \backslash S$ are thin and dashed.

becomes seemingly simpler. Since the embedding of $G$ is fixed and since $G^{\prime}$ has to be planar, edges in $S$ can only be embedded inside faces of $G$. This implies that, for any two edges $e_{1}$ and $e_{2}$ that can be inserted inside a face $f$ of $G$, it is known a priori whether $e_{1}$ and $e_{2}$ can be both in $S$, namely only if their end-vertices do not alternate along the boundary of $f$. Also, $S$ can be assumed to contain only edges between vertices in distinct connected components of $G[\alpha]$, for each cluster $\alpha$, as other types of edges do not help to connect any cluster.

Consider a face $f$ of $G$ and let $\left(o_{1}, \ldots, o_{k}\right)$ be the clockwise order of the occurrences of vertices along the boundary of $f$, where $o_{i}$ and $o_{j}$ might be occurrences of the same vertex $u$ (this might happen if $u$ is a cut-vertex of $G$ ). A con-edge (short for connectivity-edge $)$ is a pair of occurrences $\left(o_{i}, o_{j}\right)$ of distinct vertices both belonging to a cluster $\alpha$, both incident to $f$, and belonging to different connected components of $G[\alpha]$ (see Fig. 1 (b)). If there are $\ell$ distinct pairs of occurrences of vertices $u$ and $v$ along a single face $f$, then there are $\ell$ con-edges connecting $u$ and $v$ in $f$, one for each pair of occurrences. A con-edge for $\alpha$ is a con-edge connecting vertices in a cluster $\alpha$. Two con-edges $e$ and $e^{\prime}$ in $f$ have a conflict or cross (we write $e \otimes e^{\prime}$ ) if the occurrences in $e$ alternate with the occurrences in $e^{\prime}$ along the boundary of $f$.

The multigraph $A$ of the con-edges is an embedded multigraph that is defined as follows. Starting from $G$, insert all the con-edges inside the faces of $G$; then, for each cluster $\alpha$ and for each connected component $G_{i}[\alpha]$ of $G[\alpha]$, contract $G_{i}[\alpha]$ into a single vertex; finally, remove all the edges of $G$. See Fig. 1 (c). With a slight abuse of notation, we denote by $A$ both the multigraph of the con-edges and the set of its edges. For each cluster $\alpha$, we denote by $A[\alpha]$ the subgraph of $A$ induced by the con-edges for $\alpha$. A planar set of spanning trees for $A$ is a set $S \subseteq A$ such that: (i) for each cluster $\alpha$, the subset $S[\alpha]$ of $S$ induced by the con-edges for $\alpha$ is a tree that spans the vertices belonging to $\alpha$; and (ii) there exist no two edges in $S$ that have a conflict. See Fig.11(d). The PSSTTM problem asks whether a planar set of spanning trees for $A$ exists.

The following lemma relates the $c$-planarity problem for embedded flat clustered graphs to the PSSTTM problem.

Lemma 1 ( [11]). An embedded flat clustered graph $C(G, T)$ is c-planar if and only if: (1) $G$ is planar; (2) there exists a face $f$ in $G$ such that when $f$ is chosen as outer face for $G$ no cycle composed of vertices of the same cluster encloses a vertex of a different cluster; and (3) a planar set of spanning trees for A exists. 


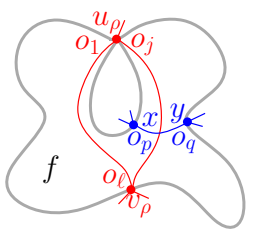

(a)

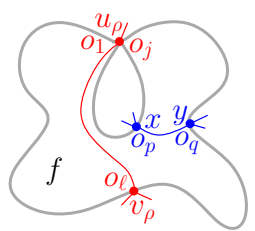

(b)

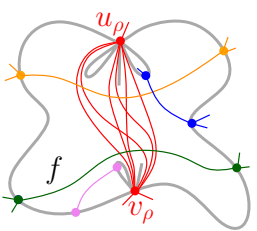

(c)

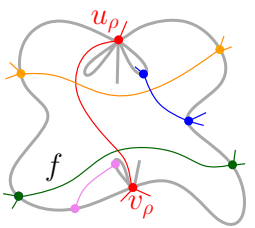

(d)

Fig. 2. Illustration for the reduction to a multigraph of the con-edges satisfying Property 1

We now introduce the concept of conflict graph $K_{A}$, which is defined as follows. Graph $K_{A}$ has a vertex for each con-edge in $A$ and has an edge $\left(e, e^{\prime}\right)$ if $e \otimes e^{\prime}$. In the remainder of the paper we will show how to decide whether a set of planar spanning trees for $A$ exists by assuming that the following property holds for $A$.

Property 1. No two con-edges for the same cluster belong to the same connected component of $K_{A}$.

We now show that $A$ can be assumed to satisfy Property 1 since $C(G, T)$ has at most two vertices per cluster on each face. Consider any face $f$ of $G$ and any cluster $\varrho$ that has two vertices $u_{\varrho}$ and $v_{\varrho}$ incident to $f$. No con-edge for $\varrho$ that connects a pair of vertices different from $\left(u_{\varrho}, v_{\varrho}\right)$ is in the connected component of $K_{A}$ containing $\left(u_{\varrho}, v_{\varrho}\right)$, given that no vertex of $\varrho$ different from $u_{\varrho}$ and $v_{\varrho}$ is incident to $f$. However, it might be the case that several con-edges $\left(u_{\varrho}, v_{\varrho}\right)$ are in the same connected component of $K_{A}$, which happens if $u_{\varrho}$, or $v_{\varrho}$, or both have several occurrences on the boundary of $f$. We show a simple reduction that gets rid of these multiple con-edges.

Let $\left(o_{1}, \ldots, o_{k}\right)$ be the clockwise order of the occurrences of vertices along $f$. Let $o_{1}, o_{j}$, and $o_{\ell}$ be occurrences of $u_{\varrho}, u_{\varrho}$, and $v_{\varrho}$, respectively, with $1<j<\ell \leq k$. Suppose that $o_{p}$ and $o_{q}$ are occurrences of vertices $x$ and $y$ in a cluster $\tau \neq \varrho$, for some $1<p<j<q<\ell$, as in Fig. 2(a). Then, all the con-edges $(x, y)$ have a conflict with con-edge $e_{\varrho}=\left(o_{j}, o_{\ell}\right)$; moreover, the con-edges $(x, y)$ form a separating set for $A[\tau]$, hence any planar set $S$ of spanning trees for $A$ contains one of them. Thus, $e_{\varrho} \notin S$ and $e_{\varrho}$ can be removed from $A$, as in Fig. 2(b). Similar reductions can be performed if $\ell<q \leq k$ and by exchanging the roles of $u_{\varrho}$ and $v_{\varrho}$. If no two occurrences $o_{p}$ and $o_{q}$ as above exist, then all the con-edges $\left(u_{\varrho}, v_{\varrho}\right)$ left cross the same set of con-edges for clusters different from $\varrho$ (see Fig. $2(\mathrm{c}))$. Hence, a single edge $\left(u_{\varrho}, v_{\varrho}\right)$ can be kept in $A$, and all the other con-edges $\left(u_{\varrho}, v_{\varrho}\right)$ can be removed from $A$ (see Fig. $2(\mathrm{~d})$ ). After repeating this reduction for all the con-edges in $A$, an equivalent instance $A$ is eventually obtained in which Property 1 is satisfied. Observe that the described simplification can be easily performed in $O\left(|C|^{2}\right)$ time. Thus, we get the following:

Lemma 2. Assume that the PSSTTM problem can be solved in $f(|A|)$ time for instances satisfying Property 11 Then the c-planarity of any embedded flat clustered graph $C$ with at most two vertices per cluster on each face can be tested in $O\left(f(|A|)+|C|^{2}\right)$ time.

Proof. Consider any embedded flat clustered graph $C$ with at most two vertices per cluster on each face. Conditions (1) and (2) in Lemma 1 can be tested in $O(|C|)$ time 
(see [11]); hence, testing the $c$-planarity of $C$ is equivalent to solve the PSSTTM problem for $A$. Finally, as described before the lemma, there exists an $O\left(|C|^{2}\right)$-time algorithm that modifies multigraph $A$ so that it satisfies Property 1

\section{Algorithm Outline}

In this section we give an outline of our algorithm for testing the existence of a planar set $S$ of spanning trees for $A$. We assume that $A$ satisfies Property 1 .

Our algorithm repeatedly tries to detect certain substructures in $A$. When it does find one of such substructures, the algorithm either "simplifies" $A$ or concludes that $A$ does not admit any planar set of spanning trees. For example, if a cluster $\alpha$ exists such that $A[\alpha]$ is not connected, then the algorithm concludes that no planar set of spanning trees exists and terminates; as another example, if conflicting con-edges $e_{\alpha}$ and $e_{\beta}$ for clusters $\alpha$ and $\beta$ exist in $A$ such that $e_{\alpha}$ is a bridge for $A[\alpha]$, then the algorithm determines that $e_{\alpha}$ has to be in $S$ and that $e_{\beta}$ can be assumed not to be in $S$.

If the algorithm determines that certain edges have to be in $S$ or can be assumed not to be in $S$, these edges are contracted or removed, respectively. Given a set $A^{\prime} \subseteq A$, the operation of removing $A^{\prime}$ from $A$ consists of updating $A:=A \backslash A^{\prime}$. Given a set $A^{\prime} \subseteq A$, the operation of contracting the edges in $A^{\prime}$ consists of identifying the endvertices of each con-edge $e$ in $A^{\prime}$ (all the con-edges different from $e$ and incident to the end-vertices of $e$ remain in $A$ ), and of updating $A:=A \backslash A^{\prime}$.

Edges are removed or contracted only when this does not alter the possibility of finding a planar set of spanning trees for $A$. Also, contractions are only applied to con-edges that cross no other con-edges; hence, after any contraction, graph $K_{A}$ only changes for the removal of the isolated vertices corresponding to the contracted edges.

As a consequence of a removal or of a contraction operation, the number of edges in $A$ decreases, that is, $A$ is "simplified". After any simplification due to the detection of a certain substructure in $A$, the algorithm will run again all previous tests for the detection of the other substructures. In fact, it is possible that a certain substructure arises from performing a simplification on $A$ (e.g., a bridge might be present in $A$ after a set of edges has been removed from $A$ ). Since detecting each substructure that leads to a simplification in $A$ can be performed in quadratic time, and since the initial size of $A$ is linear in the size of $C$, the algorithm has a cubic running time.

If none of the four tests (called TEST 1-4) and none of the eight simplifications (called SimplificAtion 1-8) described in Section 4 applies to $A$, then $A$ is a singleconflict multigraph. That is, each con-edge in $A$ crosses at most one con-edge in $A$. A linear-time algorithm for deciding the existence of a planar set of spanning trees in a single-conflict multigraph $A$ is known [11]. Hence, our algorithm uses that algorithm [11] to conclude the test of the existence of a planar set of spanning trees in $A$.

\section{Algorithm}

To ease the reading and avoid text duplication, when introducing a new lemma we always assume, without making it explicit, that all the previously defined simplifications 
do not apply, and that all the previously defined tests fail. Also, we do not make explicit the removal and contraction operations that we perform, as they straight-forwardly follow from the statement of each lemma. We start with the following test.

Lemma 3 (TEST 1). Let $\alpha$ be a cluster such that $A[\alpha]$ is disconnected. Then, there exists no planar set $S$ of spanning trees for $A$.

Proof. No set $S \subseteq A$ is such that $S[\alpha]$ induces a tree spanning the vertices in $\alpha$.

We continue with the following simplification.

Lemma 4 (SIMPLIFICATION 1). Let e be a bridge of $A[\alpha]$. Then, for every planar set $S$ of spanning trees for $A$, we have $e \in S$.

Proof. Graph $A[\alpha] \backslash\{e\}$ is disconnected; hence, by Lemma 3, no planar set of spanning trees for $A$ exists with $e \notin S$.

The following lemma is used massively in the remainder of the paper.

Lemma 5. Let $e_{\alpha}, e_{\beta} \in A$ be con-edges such that $e_{\alpha} \otimes e_{\beta}$. Let $S$ be a planar set of spanning trees for $A$ and suppose that $e_{\alpha} \notin S$. Then, $e_{\beta} \in S$.

Proof sketch. If $S$ contains neither $e_{\alpha}$ nor $e_{\beta}$, then the two paths in $S$ connecting the end-vertices of $e_{\alpha}$ and connecting the end-vertices of $e_{\beta}$ cross, a contradiction.

The algorithm continues with the following test.

Lemma 6 (TEST 2). If the conflict graph $K_{A}$ is not bipartite, then there exists no planar set $S$ of spanning trees for $A$.

Proof sketch. If an odd cycle $\mathcal{C}$ exists in $K_{A}$, then by repeated applications of Lemma 5 and of the fact that $S$ does not contain two conflicting edges, we get that any edge of $\mathcal{C}$ simultaneously should be in $S$ and should not be in $S$, a contradiction.

The contraction of con-edges chosen to be in $S$ might lead to self-loops in $A$, a situation that is handled in the following.

Lemma 7 (SIMPLIFICATION 2). Let $e \in A$ be a self-loop. Then, for every planar set $S$ of spanning trees for $A$, we have $e \notin S$.

Proof. Since a tree does not contain any self-loop, the lemma follows.

Con-edges that do not cross any other con-edge can be safely chosen to be in $S$.

Lemma 8 (SimplificATION 3). Let e be any con-edge in A that does not have a conflict with any other con-edge in A. Then, there exists a planar set $S$ of spanning trees for $A$ if and only if there exists a planar set $S^{\prime}$ of spanning trees for $A$ such that $e \in S^{\prime}$.

Proof sketch. Let $S$ be any planar set of spanning trees for $A$. If $e \notin S$, then $S \cup\{e\}$ contains a cycle $\mathcal{C}$ of con-edges for the same cluster. Let $e^{\prime}$ be any edge of $\mathcal{C}$ different from $e$. Then, $S^{\prime}=S \cup\{e\} \backslash\left\{e^{\prime}\right\}$ is a planar set of spanning trees for $A$. 


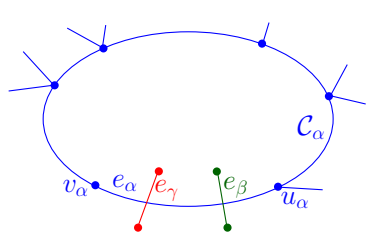

(a)

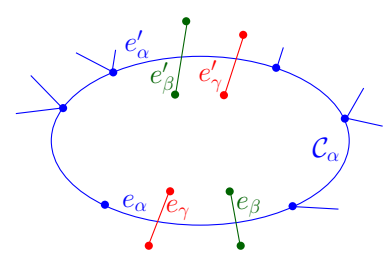

(b)

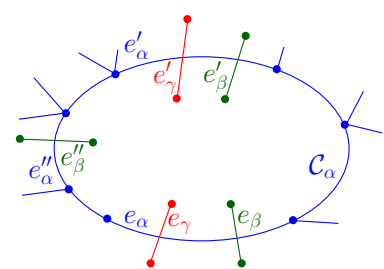

(c)

Fig. 3. The setting for (a) Lemma 9 (b) Lemma 10, and (c) Lemma 11

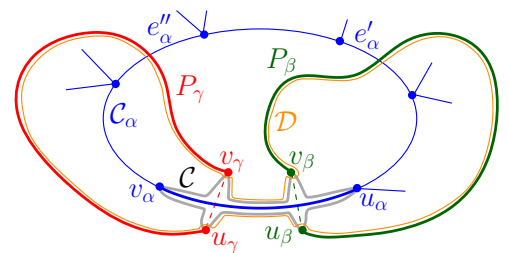

Fig. 4. Illustration for the proof of Lemma 9

In the next three lemmata we deal with the following setting. Assume that there exist con-edges $e_{\alpha}, e_{\beta}, e_{\gamma} \in A$ for distinct clusters $\alpha, \beta$, and $\gamma$, respectively, such that $e_{\alpha} \otimes e_{\beta}$ and $e_{\alpha} \otimes e_{\gamma}$. Since TEST 2 fails on $A, e_{\beta}$ does not cross $e_{\gamma}$. Let $\mathcal{C}_{\alpha}$ be any of the two facial cycles of $A[\alpha]$ incident to $e_{\alpha}$, where a facial cycle of $A[\alpha]$ is a simple cycle all of whose edges appear on the boundary of a single face of $A[\alpha]$. Assume w.l.o.g. that $e_{\alpha}$ is crossed first by $e_{\beta}$ and then by $e_{\gamma}$ when $\mathcal{C}_{\alpha}$ is traversed clockwise. See Fig. 3. (a).

The next lemma presents a condition in which we can delete $e_{\alpha}$ from $A$.

Lemma 9 (SIMPLIFICATION 4). Suppose that there exists no con-edge of $\mathcal{C}_{\alpha}$ different from $e_{\alpha}$ that has a conflict with both a con-edge for $\beta$ and a con-edge for $\gamma$. Then, for every planar set $S$ of spanning trees for $A$, we have $e_{\alpha} \notin S$.

Proof sketch. See Fig. 4. Let $u_{\alpha}$ and $v_{\alpha}\left(u_{\beta}\right.$ and $v_{\beta}, u_{\gamma}$ and $\left.v_{\gamma}\right)$ be the end-vertices of $e_{\alpha}$ (resp. of $e_{\beta}$, of $e_{\gamma}$ ). By Property 1, a closed simple curve $\mathcal{C}$ can be drawn through $u_{\alpha}, u_{\beta}, u_{\gamma}, v_{\alpha}, v_{\gamma}$, and $v_{\beta}$, with $e_{\alpha}, e_{\beta}$, and $e_{\gamma}$ in its interior and every other con-edge for $\alpha, \beta$, and $\gamma$ in its exterior. If $e_{\alpha} \in S$, then $e_{\beta}, e_{\gamma} \notin S$. Then, the path $P_{\beta}$ in $S$ connecting $u_{\beta}$ and $v_{\beta}$ and the path $P_{\gamma}$ in $S$ connecting $u_{\gamma}$ and $v_{\gamma}$ cross $\mathcal{C}_{\alpha}$ at different edges $e_{\alpha}^{\prime}$ and $e_{\alpha}^{\prime \prime}$. Hence, the end-vertices of $e_{\alpha}^{\prime}$ are on different sides of the cycle $\mathcal{D}$ composed of $P_{\beta}$, of $P_{\gamma}$, and of the paths in $\mathcal{C}$ between $u_{\beta}$ and $u_{\gamma}$ and between $v_{\beta}$ and $v_{\gamma}$. However, no con-edge for $\alpha$ in $S$ crosses $\mathcal{D}$, hence $S$ does not connect $\alpha$.

The next two lemmata state conditions in which no planar set of spanning trees for $A$ exists. Their statements are illustrated in Figs. 3 (b) and 3 (c), respectively; further, they can be proved with arguments similar to the ones in the proof of Lemma 9 .

Lemma 10 (TEST 3). Suppose that there exist con-edges $e_{\alpha}^{\prime}, e_{\beta}^{\prime}, e_{\gamma}^{\prime} \in A$ for clusters $\alpha, \beta$, and $\gamma$, respectively, such that $e_{\alpha}^{\prime} \neq e_{\alpha}$, $e_{\alpha}^{\prime}$ belongs to $\mathcal{C}_{\alpha}$, and $e_{\alpha}^{\prime} \otimes e_{\beta}^{\prime}$ as well 


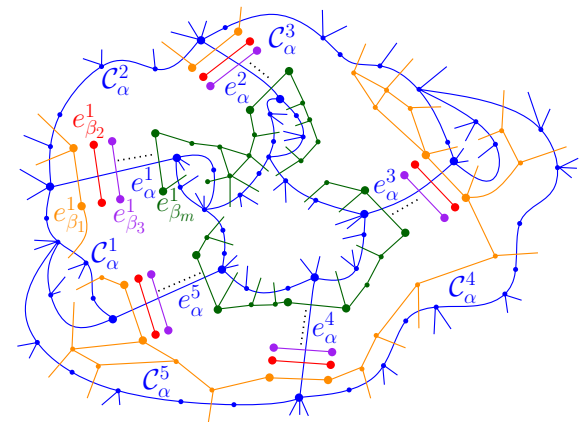

Fig. 5. A partial representation of the $\alpha$-donut for $e_{\alpha}$

as $e_{\alpha}^{\prime} \otimes e_{\gamma}^{\prime}$. Assume that $e_{\alpha}^{\prime}$ is crossed first by $e_{\beta}^{\prime}$ and then by $e_{\gamma}^{\prime}$ when $\mathcal{C}_{\alpha}$ is traversed clockwise. Then, no planar set of spanning trees for A exists.

Lemma 11 (TEST 4). Suppose that con-edges $e_{\alpha}^{\prime}, e_{\alpha}^{\prime \prime} \in A$ for $\alpha$ exist in $\mathcal{C}_{\alpha}$, and such that $e_{\alpha}, e_{\alpha}^{\prime \prime}$, and $e_{\alpha}^{\prime}$ occur in this order along $\mathcal{C}_{\alpha}$, when clockwise traversing $\mathcal{C}_{\alpha}$. Suppose also that there exist con-edges $e_{\beta}^{\prime}, e_{\beta}^{\prime \prime} \in A$ for $\beta$ and $e_{\gamma}^{\prime} \in A$ for $\gamma$ such that $e_{\alpha}^{\prime} \otimes e_{\beta}^{\prime}$, $e_{\alpha}^{\prime} \otimes e_{\gamma}^{\prime}$, and $e_{\alpha}^{\prime \prime} \otimes e_{\beta}^{\prime \prime}$. Then, no planar set of spanning trees for $A$ exists.

If Simplifications 1-4 do not apply to $A$ and Tests $1-4$ fail on $A$, then the conedges for a cluster $\alpha$ that are crossed by con-edges for (at least) two other clusters have a nice structure, that we call $\alpha$-donut (see Fig. 5). Consider a con-edge $e_{\alpha} \in A$ for $\alpha$ crossing con-edges $e_{\beta_{1}}, \ldots, e_{\beta_{m}}$ for clusters $\beta_{1}, \ldots, \beta_{m}$, with $m \geq 2$. An $\alpha$-donut for $e_{\alpha}$ consists of a sequence $e_{\alpha}^{1}, \ldots, e_{\alpha}^{k}, e_{\alpha}^{k+1}$ of con-edges for $\alpha$ with $k \geq 2$, called spokes of the $\alpha$-donut, of a sequence $\mathcal{C}_{\alpha}^{1}, \ldots, \mathcal{C}_{\alpha}^{k}, \mathcal{C}_{\alpha}^{k+1}=\mathcal{C}_{\alpha}^{1}$ of facial cycles in $A[\alpha]$, and of sequences $e_{\beta_{j}}^{1}, \ldots, e_{\beta_{j}}^{k}$ of con-edges for $\beta_{j}$, for each $1 \leq j \leq m$, such that the following hold for every $1 \leq i \leq k$ : (a) $e_{\alpha}=e_{\alpha}^{1}$; (b) $e_{\alpha}^{i} \otimes e_{\beta_{j}}^{i}$, for every $1 \leq j \leq m$; (c) $\mathcal{C}_{\alpha}^{i}$ and $\mathcal{C}_{\alpha}^{i+1}$ share edge $e_{\alpha}^{i}$; (d) edge $e_{\alpha}^{i}$ is crossed by $e_{\beta_{1}}^{i}, \ldots, e_{\beta_{m}}^{i}$ in this order when $\mathcal{C}_{\alpha}^{i}$ is traversed clockwise; (e) all the con-edges of $\mathcal{C}_{\alpha}^{i+1}$ encountered when clockwise traversing $\mathcal{C}_{\alpha}^{i+1}$ from $e_{\alpha}^{i}$ to $e_{\alpha}^{i+1}$ do not cross any con-edge for $\beta_{2}, \ldots, \beta_{m}$; and (f) all the con-edges of $\mathcal{C}_{\alpha}^{i+1}$ encountered when clockwise traversing $\mathcal{C}_{\alpha}^{i+1}$ from $e_{\alpha}^{i+1}$ to $e_{\alpha}^{i}$ do not cross any con-edge for $\beta_{1}, \ldots, \beta_{m-1}$. We have the following.

Lemma 12. For every con-edge $e_{\alpha} \in A$ for $\alpha$, there exists an $\alpha$-donut for $e_{\alpha}$.

Proof sketch. Let $e_{\alpha}^{1}=e_{\alpha}$ and let $\mathcal{C}_{\alpha}^{1}$ and $\mathcal{C}_{\alpha}^{2}$ be the facial cycles incident to $e_{\alpha}^{1}$. Since SimplificAtion 1 does not apply to $A, \mathcal{C}_{\alpha}^{1} \neq \mathcal{C}_{\alpha}^{2}$. Let $e_{\beta_{1}}^{1}, \ldots, e_{\beta_{m}}^{1}$ be the conedges for $\beta_{1}, \ldots, \beta_{m}$, respectively, ordered as they cross $e_{\alpha}^{1}$ when clockwise traversing $\mathcal{C}_{\alpha}^{1}$. Since SIMPLIFICATION 4 does not apply to $A$ and TESTS 3-4 fail on $A$, a con-edge $e_{\alpha}^{2}$ exists in $\mathcal{C}_{\alpha}^{2}$ that is crossed by con-edges $e_{\beta_{1}}^{2}, \ldots, e_{\beta_{m}}^{2}$ for $\beta_{1}, \ldots, \beta_{m}$, respectively, in this order when clockwise traversing $\mathcal{C}_{\alpha}^{2}$; further, all the con-edges of $\mathcal{C}_{\alpha}^{2}$ encountered when clockwise traversing $\mathcal{C}_{\alpha}^{2}$ from $e_{\alpha}^{1}$ to $e_{\alpha}^{2}$ (from $e_{\alpha}^{2}$ to $e_{\alpha}^{1}$ ) do not cross any con-edges for $\beta_{2}, \ldots, \beta_{m}$ (resp. for $\beta_{1}, \ldots, \beta_{m-1}$ ). This argument is repeated for $i=3, \ldots, k$ to determine a facial cycle $\mathcal{C}_{\alpha}^{i}$ containing $e_{\alpha}^{i-1}$ and to determine edges $e_{\alpha}^{i}, e_{\beta_{1}}^{i}, \ldots, e_{\beta_{m}}^{i}$. 
Eventually, facial cycle $\mathcal{C}_{\alpha}^{k+1}=\mathcal{C}_{\alpha}^{1}$ of $A[\alpha]$ is considered in which the two con-edges that are crossed by con-edges for all of $\beta_{1}, \ldots, \beta_{m}$ are $e_{\alpha}^{k}$ and $e_{\alpha}^{1}$.

The $\alpha$-donut for $e_{\alpha}$ can be computed efficiently. Further, we have the following lemma, whose proof is similar to the one of Lemma 9

Lemma 13. Let $e_{\alpha}^{1}, \ldots, e_{\alpha}^{k}$ be the spokes of the $\alpha$-donut for $e_{\alpha}$. Then, if a planar set $S$ of spanning trees for A exists, it contains exactly one of $e_{\alpha}^{1}, \ldots, e_{\alpha}^{k}$.

Consider a con-edge $e$ for a cluster $\alpha$. The conflicting structure $M(e)$ of $e$ is a sequence of sets $H_{0}(e), L_{1}(e), H_{1}(e), L_{2}(e), H_{2}(e), \ldots$ of con-edges which correspond to the layers of a BFS traversal starting at $e$ of the connected component of $K_{A}$ containing $e$. That is: $H_{0}(e)=\{e\}$; then, for $i \geq 1, L_{i}(e)$ is the set of con-edges that cross con-edges in $H_{i-1}(e)$ and that are not in $L_{i-1}(e)$, and $H_{i}(e)$ is the set of con-edges that cross con-edges in $L_{i}(e)$ and that are not in $H_{i-1}(e)$.

We now study the conflicting structures of the spokes $e_{\alpha}^{1}, \ldots, e_{\alpha}^{k}$ of the $\alpha$-donut of a con-edge $e_{\alpha}$ for $\alpha$. No two edges in a set $H_{i}\left(e_{\alpha}\right)$ or in a set $L_{i}\left(e_{\alpha}\right)$ have a conflict, as otherwise TEST 2 would succeed. Also, by Lemma 5 any planar set $S$ of spanning trees for $A$ contains either all the edges in $\bigcup_{i} H_{i}\left(e_{\alpha}\right)$ or all the edges in $\bigcup_{i} L_{i}\left(e_{\alpha}\right)$.

Assume that $e_{\alpha}$ has a conflict with at least two con-edges for other clusters. For any $1 \leq i \leq k$, we say that $e_{\alpha}^{i}$ and $e_{\alpha}^{i+1}$ have isomorphic conflicting structures if they belong to isomorphic connected components of $K_{A}$ and if the vertices of these components that are in correspondence under the isomorphism represent con-edges for the same cluster. Formally, $e_{\alpha}^{i}$ and $e_{\alpha}^{i+1}$ have isomorphic conflicting structures if there exists a bijective mapping $\delta$ from the edges in $M\left(e_{\alpha}^{i}\right)$ to the edges in $M\left(e_{\alpha}^{i+1}\right)$ such that: (1) $e$ is a con-edge for a cluster $\varrho$ if and only if $\delta(e)$ is a con-edge for $\varrho$, for every $e \in M\left(e_{\alpha}^{i}\right)$; (2) $e \in H_{j}\left(e_{\alpha}^{i}\right)$ if and only if $\delta(e) \in H_{j}\left(e_{\alpha}^{i+1}\right)$, for every $e \in M\left(e_{\alpha}^{i}\right)$; (3) $e \in L_{j}\left(e_{\alpha}^{i}\right)$ if and only if $\delta(e) \in L_{j}\left(e_{\alpha}^{i+1}\right)$, for every $e \in M\left(e_{\alpha}^{i}\right)$; and (4) $e \otimes f$ if and only if $\delta(e) \otimes \delta(f)$, for every $e, f \in M\left(e_{\alpha}^{i}\right)$. Observe that the isomorphism of two conflicting structures can be tested efficiently.

We will prove in the following four lemmata that by examining the conflicting structures for the spokes of the $\alpha$-donut for $e_{\alpha}$, a decision on whether some spoke is or is not in $S$ can be taken without loss of generality. We start with the following:

Lemma 14 (Simplification 5). Suppose that spokes $e_{\alpha}^{i}$ and $e_{\alpha}^{i+1}$ have isomorphic conflicting structures. Then, there exists a planar set $S$ of spanning trees for $A$ if and only if there exists a planar set $S^{\prime}$ of spanning trees for A such that $e_{\alpha}^{i} \notin S^{\prime}$.

Proof sketch. Suppose that a planar set $S$ of spanning trees for $A$ exists with $e_{\alpha}^{i} \in S$. By Lemma 5. $\bigcup_{j} H_{j}\left(e_{\alpha}^{i}\right) \subseteq S$ and $S \cap \bigcup_{j} L_{j}\left(e_{\alpha}^{i}\right)=\emptyset$. By Lemma 13, $e_{\alpha}^{i+1} \notin S$, hence $\bigcup_{j} L_{j}\left(e_{\alpha}^{i+1}\right) \subseteq S$ and $S \cap \bigcup_{j} H_{j}\left(e_{\alpha}^{i+1}\right)=\emptyset$. Let $S^{\prime}$ be the set of con-edges obtained from $S$ by removing $\bigcup_{j} H_{j}\left(e_{\alpha}^{i}\right)$ and $\bigcup_{j} L_{j}\left(e_{\alpha}^{i+1}\right)$ and by adding $\bigcup_{j} L_{j}\left(e_{\alpha}^{i}\right)$ and $\bigcup_{j} H_{j}\left(e_{\alpha}^{i+1}\right)$. The lemma follows from the claim that $S^{\prime}$ is a planar set of spanning trees for $A$. The proof for this claim consists of two parts. In the first one, it is shown that no two con-edges in $S^{\prime}$ cross, by exploiting the absence of crossings in $S$ and the properties of $M\left(e_{\alpha}^{i}\right)$ and $M\left(e_{\alpha}^{i+1}\right)$. In the second one, it is shown that, for each cluster $\mu$, the graph induced by the con-edges in $S^{\prime}[\mu]$ is a tree that spans the vertices in $\mu$; this 
proof uses topological arguments to establish that the only con-edge for $\mu$ in $S^{\prime} \backslash S$ has its end-vertices in different connected components of the graph obtained from $S[\mu]$ by removing the only con-edge for $\mu$ in $S \backslash S^{\prime}$.

Next, we study non-isomorphic spokes. Let $e_{\alpha}^{i}$ be a spoke of the $\alpha$-donut for $e_{\alpha}$. Assume that $L_{1}\left(e_{\alpha}^{i}\right)$ contains a con-edge $e_{\beta}^{i}$ for a cluster $\beta$, and that $H_{1}\left(e_{\alpha}^{i}\right)$ contains a con-edge $e_{\gamma}^{i}$ for a cluster $\gamma$, where $e_{\alpha}^{i} \otimes e_{\beta}^{i}$ and $e_{\beta}^{i} \otimes e_{\gamma}^{i}$. By Property 1 since $e_{\gamma}^{i}$ and $e_{\alpha}^{i}$ belong to the same connected component of $K_{A}$ and do not cross (as otherwise TEST 2 would succeed), it follows that $e_{\gamma}^{i}$ does not cross any con-edge for $\alpha$, hence it lies in one of the two faces $f_{\alpha}^{i}$ and $f_{\alpha}^{i+1}$ of $A[\alpha]$ that $e_{\alpha}^{i}$ shares with spokes $e_{\alpha}^{i-1}$ and $e_{\alpha}^{i+1}$, respectively. Assume w.l.o.g. that $e_{\gamma}^{i}$ lies in $f_{\alpha}^{i+1}$. By Lemma 12 $L_{1}\left(e_{\alpha}^{i+1}\right)$ contains a con-edge $e_{\beta}^{i+1}$ for $\beta$, where $e_{\alpha}^{i+1} \otimes e_{\beta}^{i+1}$.

The next two lemmata discuss the case in which $M\left(e_{\alpha}^{i+1}\right)$ contains a con-edge for $\gamma$ that has a conflict with $e_{\beta}^{i+1}$ and the case in which it does not. We start with the latter.

Lemma 15 (SIMPLIFICATION 6). Suppose that no con-edge $e_{\gamma}^{i+1}$ for $\gamma$ exists such that $e_{\gamma}^{i+1} \otimes e_{\beta}^{i+1}$, and that a planar set $S$ of spanning trees for $A$ exists. Then, $e_{\alpha}^{i} \in S$.

Proof sketch. If a planar set $S$ of spanning trees for $A$ exists with $e_{\alpha}^{i} \notin S$, then by Lemma 5 we have $e_{\beta}^{i} \in S$ and $e_{\gamma}^{i} \notin S$. Then, the paths $P_{\alpha}^{i}$ and $P_{\gamma}^{i}$ connecting the end-vertices of $e_{\alpha}^{i}$ and $e_{\gamma}^{i}$, together with a closed simple curve $\mathcal{C}$ surrounding $e_{\alpha}^{i}, e_{\beta}^{i}$, and $e_{\gamma}^{i}$ form a closed curve $\mathcal{D}$ that contains vertices in $\beta$ on both sides. However, $\mathcal{D}$ cannot be crossed by any con-edge for $\beta$ in $S$, thus $S$ does not connect $\beta$.

Lemma 16 (SIMPLIFICATION 7). Suppose that a con-edge $e_{\gamma}^{i+1}$ for $\gamma$ exists with $e_{\gamma}^{i+1} \otimes$ $e_{\beta}^{i+1}$. If a planar set $S$ of spanning trees for A exists, then either $e_{\alpha}^{i} \in S$ or $e_{\alpha}^{i+1} \in S$.

Proof sketch. By Lemma 13, at most one out of $e_{\alpha}^{i}$ and $e_{\alpha}^{i+1}$ belongs to $S$. To prove that at least one out of $e_{\alpha}^{i}$ and $e_{\alpha}^{i+1}$ belongs to $S$, by Lemma 5 it suffices to prove that at most one out of $e_{\beta}^{i}$ and $e_{\beta}^{i+1}$ belongs to $S$. This is accomplished again by Lemma 13 and by proving that $e_{\beta}^{i+1}$ is a spoke of the $\beta$-donut for $e_{\beta}^{i}$.

Observe that Simplification 7 can be applied in the case in which the $\alpha$-donut for $e_{\alpha}$ has at least three spokes. Namely, in that case, by Lemmata 13 and 16 all the spokes different from $e_{\alpha}^{i}$ and $e_{\alpha}^{i+1}$ can be removed from $A$.

Next, assume that there exists an $\alpha$-donut with exactly two spokes $e_{\alpha}^{1}$ and $e_{\alpha}^{2}$. Consider the smallest $j \geq 1$ such that one of the following holds:

(1) there exist con-edges $e_{\mu} \in L_{j}\left(e_{\alpha}^{a}\right)$ and $e_{\nu} \in H_{j-1}\left(e_{\alpha}^{a}\right)$ for clusters $\mu$ and $\nu$, resp., such that $e_{\mu} \otimes e_{\nu}$, and there exists no con-edge $g_{\mu} \in L_{j}\left(e_{\alpha}^{b}\right)$ for $\mu$ such that $g_{\mu} \otimes g_{\nu}$ with $g_{\nu}$ con-edge for $\nu$ in $H_{j-1}\left(e_{\alpha}^{b}\right)$, for some $a, b \in\{1,2\}$ with $a \neq b$; or

(2) there exist con-edges $e_{\mu} \in H_{j}\left(e_{\alpha}^{a}\right)$ and $e_{\nu} \in L_{j}\left(e_{\alpha}^{a}\right)$ for clusters $\mu$ and $\nu$, resp., such that $e_{\mu} \otimes e_{\nu}$, and there exists no con-edge $g_{\mu} \in H_{j}\left(e_{\alpha}^{b}\right)$ for $\mu$ such that $g_{\mu} \otimes g_{\nu}$ with $g_{\nu}$ con-edge for $\nu$ in $L_{j}\left(e_{\alpha}^{b}\right)$, for some $a, b \in\{1,2\}$ with $a \neq b$. We have the following.

Lemma 17 (SimplificATION 8). Assume that a planar set $S$ of spanning trees for $A$ exists. Then, $e_{\mu} \in S$. 
Proof sketch. The proof uses topological arguments to establish the following claim: If $e_{\mu} \notin S$, then the end-vertices of $e_{\mu}$ are on diffent sides of a cycle composed of conedges for $\nu$ that cannot be crossed by con-edges for $\mu$ in $S$, hence $S$ does not connect $\mu$, a contradiction.

We now prove that our simplifications form a "complete set".

Lemma 18. Suppose that SimplificATIOns 1-8 do not apply to $A$ and that TeSTS 1-4 fail on A. Then, every con-edge in A crosses exactly one con-edge in A.

Proof sketch. Since SimplifiCATIONS 2-3 do not apply to $A$, every con-edge crosses at least one con-edge. Suppose, for a contradiction, that there exists a con-edge for a cluster $\alpha$ that has a conflict with at least two con-edges. Since SimplifiCATIONS 1-4 do not apply to $A$ and TESTS $1-4$ fail on $A$, by Lemma 12 there exists an $\alpha$-donut with spokes $e_{\alpha}^{1}, \ldots, e_{\alpha}^{k}$. If the conflicting structures of $e_{\alpha}^{1}$ and $e_{\alpha}^{2}$ are isomorphic, then SimPLIFICATION 5 applies to $A$. Otherwise, if $k \geq 3$ and the conflicting structures of $e_{\alpha}^{1}$ and $e_{\alpha}^{2}$ are isomorphic (not isomorphic) when restricted to sets $H_{0}\left(e_{\alpha}^{j}\right), L_{1}\left(e_{\alpha}^{j}\right)$, and $H_{1}\left(e_{\alpha}^{j}\right)$, then Simplification 7 (resp. Simplification 6) applies to $A$. If $k=2$ and the conflicting structures of $e_{\alpha}^{1}$ and $e_{\alpha}^{2}$ are not isomorphic, then SIMPLIFICATION 8 applies to $A$. This provides a contradiction.

A linear-time algorithm to determine whether a planar set $S$ of spanning trees exists for a single-conflict graph is known [11]. We thus finally get:

Theorem 1. There exists an $O\left(|C|^{3}\right)$-time algorithm to test the c-planarity of an embedded flat clustered graph $C$ with at most two vertices per cluster on each face.

Proof. Multigraph $A$ can be easily constructed in $O\left(|C|^{2}\right)$ time, so that $A$ has $O(|C|)$ vertices and edges and satisfies Property 1 By Lemma 2] it suffices to show how to solve the PSSTTM problem for $A$ in $O\left(|C|^{3}\right)$ time. Algorithm 1 correctly determines whether a planar set $S$ of spanning trees for $A$ exists, by Lemmata $3-18$. It can be easily tested in $O\left(|A|^{2}\right)$ time whether the pre-conditions of each of SIMPLIFICATIONS 1-8 and TESTS $1-4$ are satisfied; also, the actual simplifications, that is, removing and contracting edges in $A$, can be performed in $O(|A|)$ time. Furthermore, the algorithm in [11] runs in $O(|A|)$ time. Since the number of performed tests and simplifications is in $O(|A|)$, the total running time is in $O\left(|A|^{3}\right)$, and hence in $O\left(|C|^{3}\right)$.

\section{Conclusions}

We presented a polynomial-time algorithm for testing $c$-planarity of embedded flat clustered graphs with at most two vertices per cluster on each face. An interesting extension of our results would be to devise an FPT algorithm to test $c$-planarity of embedded flat clustered graphs, where the parameter is the maximum number $k$ of vertices of the same cluster on any face. Several key lemmata (e.g. Lemmata 5 and 6 ) do not apply if $k>2$, hence even an algorithm with running time $n^{O(f(k))}$ seems to be an elusive goal.

\section{References}

1. Angelini, P., Di Battista, G., Frati, F., Jelínek, V., Kratochvíl, J., Patrignani, M., Rutter, I.: Testing planarity of partially embedded graphs. In: SODA 2010, pp. 202-221. ACM (2010) 
2. Angelini, P., Frati, F., Patrignani, M.: Splitting clusters to get C-planarity. In: Eppstein, D., Gansner, E.R. (eds.) GD 2009. LNCS, vol. 5849, pp. 57-68. Springer, Heidelberg (2010)

3. Bertolazzi, P., Di Battista, G., Liotta, G., Mannino, C.: Upward drawings of triconnected digraphs. Algorithmica 12(6), 476-497 (1994)

4. Chimani, M., Di Battista, G., Frati, F., Klein, K.: Advances on testing c-planarity of embedded flat clustered graphs. CoRR, abs/1408.2595 (2014)

5. Chimani, M., Gutwenger, C., Jansen, M., Klein, K., Mutzel, P.: Computing maximum cplanar subgraphs. In: Tollis, I.G., Patrignani, M. (eds.) GD 2008. LNCS, vol. 5417, pp. 114 120. Springer, Heidelberg (2009)

6. Chimani, M., Klein, K.: Shrinking the search space for clustered planarity. In: Didimo, W., Patrignani, M. (eds.) GD 2012. LNCS, vol. 7704, pp. 90-101. Springer, Heidelberg (2013)

7. Cornelsen, S., Wagner, D.: Completely connected clustered graphs. J. Discrete Algorithms 4(2), 313-323 (2006)

8. Cortese, P.F., Di Battista, G., Frati, F., Patrignani, M., Pizzonia, M.: C-planarity of cconnected clustered graphs. J. Graph Algorithms Appl. 12(2), 225-262 (2008)

9. Cortese, P.F., Di Battista, G., Patrignani, M., Pizzonia, M.: Clustering cycles into cycles of clusters. J. Graph Alg. Appl. 9(3), 391-413 (2005)

10. Dahlhaus, E.: A linear time algorithm to recognize clustered planar graphs and its parallelization. In: Lucchesi, C.L., Moura, A.V. (eds.) LATIN 1998. LNCS, vol. 1380, pp. 239-248. Springer, Heidelberg (1998)

11. Di Battista, G., Frati, F.: Efficient c-planarity testing for embedded flat clustered graphs with small faces. J. Graph Alg. Appl. 13(3), 349-378 (2009)

12. Didimo, W., Giordano, F., Liotta, G.: Overlapping cluster planarity. J. Graph Algorithms Appl. 12(3), 267-291 (2008)

13. Feng, Q.W., Cohen, R.F., Eades, P.: Planarity for clustered graphs. In: Moore, W., Luk, W. (eds.) FPL 1995. LNCS, vol. 975, pp. 213-226. Springer, Heidelberg (1995)

14. Garg, A., Tamassia, R.: On the computational complexity of upward and rectilinear planarity testing. SIAM Journal on Computing 31(2), 601-625 (2001)

15. Goodrich, M.T., Lueker, G.S., Sun, J.Z.: C-planarity of extrovert clustered graphs. In: Healy, P., Nikolov, N.S. (eds.) GD 2005. LNCS, vol. 3843, pp. 211-222. Springer, Heidelberg (2006)

16. Gutwenger, C., Jünger, M., Leipert, S., Mutzel, P., Percan, M., Weiskircher, R.: Advances in c-planarity testing of clustered graphs. In: Goodrich, M.T., Kobourov, S.G. (eds.) GD 2002. LNCS, vol. 2528, pp. 220-235. Springer, Heidelberg (2002)

17. Jelínek, V., Jelínková, E., Kratochvíl, J., Lidický, B.: Clustered planarity: Embedded clustered graphs with two-component clusters. In: Tollis, I.G., Patrignani, M. (eds.) GD 2008. LNCS, vol. 5417, pp. 121-132. Springer, Heidelberg (2009)

18. Jelínek, V., Suchý, O., Tesař, M., Vyskočil, T.: Clustered planarity: Clusters with few outgoing edges. In: Tollis, I.G., Patrignani, M. (eds.) GD 2008. LNCS, vol. 5417, pp. 102-113. Springer, Heidelberg (2009)

19. Jelínková, E., Kára, J., Kratochvíl, J., Pergel, M., Suchý, O., Vyskocil, T.: Clustered planarity: Small clusters in cycles and Eulerian graphs. J. Graph Alg. Appl. 13(3), 379-422 (2009)

20. Kratochvíl, J., Lubiw, A., Nesetril, J.: Noncrossing subgraphs in topological layouts. SIAM J. Discrete Math. 4(2), 223-244 (1991)

21. Schaefer, M.: Toward a theory of planarity: Hanani-Tutte and planarity variants. J. Graph Algorithms Appl. 17(4), 367-440 (2013)

22. Schaeffer, S.E.: Graph clustering. Computer Science Review 1(1), 27-64 (2007)

23. Tamassia, R.: On embedding a graph in the grid with the minimum number of bends. SIAM J. Comput. 16(3), 421-444 (1987) 